\title{
Bovine tuberculosis: herd-level surveillance of Mycobacterium bovis genotypes in Northern Ireland (2003-2008)
}

R A Skuce, T R Mallon, C McCormick, S H McBride, G Clarke, A Thompson, C Cousens, A W Gordon, S W McDowell Agri-Food and Biosciences Institute, Belfast United Kingdom

Email: robin.skuce@afbini.gov.uk

Introduction Bovine TB is a respiratory disease caused by Mycobacterium bovis infection. It is the most complex and difficult multi-species, endemic disease facing government, the veterinary profession and the farming industry in the UK (Reynolds 2006). The maintenance and spread of infection within and between populations of cattle and populations of wildlife is poorly understood, with investigations hampered by difficulties in accurately defining epidemiologically-linked cases. The recent development of $M$. bovis genotyping schemes, based on recording DNA repeat copy number variation (variable number tandem repeats or VNTRs) offers highly-discriminating tools for investigating the spread of M. bovis (Skuce et al 2005, Smith et al 2006). Systematic application of M. bovis genotyping in conjunction with tracing of cattle movements and wildlife surveillance can be expected to provide valuable insights into disease source and spread. The objective of this study was to investigate, by means of structured herd-level surveillance, the genotype diversity, distribution and structure of the current $M$. bovis population.

Materials and methods New, culture-confirmed bovine TB herd incidents were enlisted on a rolling 365 day period (2003-2008). M. bovis was isolated and confirmed using standard procedures. The first (disclosing) M. bovis isolate per herd was subjected to genotyping (Skuce et al 2005). VNTR profiling, spoligotyping, nomenclature, reference isolates and quality control were as described (Skuce et al 2005). Animal-level data (movements and test records) were derived from the Animal and Public Health Information System (APHIS, Houston 2001). M. bovis isolates were geo-referenced to their final herd using MapInfo Professional v7.5. To investigate potential associations between M. bovis genotype and location and inter-annual differences in frequency, contingency tables were analysed using Chi-square tests with Monte Carlo simulation and by test for trend statistics.

Results 175 genetically-distinct $M$. bovis genotypes were identified in 8,630 isolates from 6,609 herds. On average 73 genotypes were identified every year, with 29 genotypes present in all 6 years. M. bovis genotypes showed striking geographical localisation and significant association $(\mathrm{P}<0.001)$ to regions. However, genotypes were also trans-located significant distances from their normal 'home range'. Whilst the frequency of most M. bovis genotypes was relatively stable over the survey period, significant differences $(\mathrm{P}<0.001)$ were observed for some genotypes in years 2003 to 2008 , indicating that the $M$. bovis population was not entirely static. Despite regions being dominated by geographically-localised genotypes, significant and exploitable (in outbreak investigation) local diversity was still present.

Conclusions Significant genotype diversity was disclosed in the sampled Northern Ireland M. bovis population. The population was highly geographically structured, with different $M$. bovis genotypes tending to cluster significantly in distinct regions. This suggested that most sources tended to be local and relatively stable. Whilst the frequency of most $M$. bovis genotypes was relatively stable over the survey period, expansion and contraction of some genotypes was evident. $M$. bovis genotyping, in conjunction with comprehensive cattle movement databases and wildlife surveillance, offers a powerful tool for investigating bovine TB source, maintenance and spread. The population structure and the performance characteristics of $M$. bovis genotyping support its use to answer detailed epidemiological questions of direct policy relevance.

Acknowledgements The authors gratefully acknowledge funding from the Department of Agriculture and Rural Development for Northern Ireland (Project DARD0407) and helpful discussions with Noel H Smith (VLA Weybridge).

\section{References}

Houston, R. 2001. A computerized database system for bovine traceability. Revue Scientifique et Technique 20, 652-661. Reynolds, D. 2006. A review of tuberculosis science and policy in Great Britain. Veterinary Microbiology 112, 119-126. Skuce, R. A., McDowell, S. W., Mallon, T. R., Luke, B., Breadon, E. L., Lagan, P. L., McCormick, C. M., McBride, S. H. \& Pollock, J. M. 2005. Discrimination of isolates of Mycobacterium bovis in Northern Ireland on the basis of variable numbers of tandem repeats (VNTRs). The Veterinary Record 157, 501-504.

Smith, N. H., Gordon, S. V., De La Rua-Domenech, R., Clifton-Hadley, R. S., \& Hewinson, R. G. 2006. Bottlenecks and broomsticks: the molecular evolution of Mycobacterium bovis. Nature Reviews Microbiology 4, 670-681. 